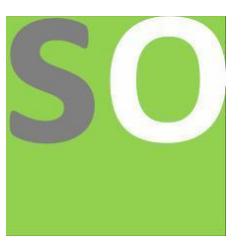

Article title: On the Relativity of the Speed of Light

Authors: XD Dongfang[1]

Affiliations: Wutong Mountain National Forest Park[1]

Orcid ids: 0000-0002-3644-5170[1]

Contact e-mail: xddongfang@gmail.com

License information: This work has been published open access under Creative Commons Attribution License $\mathrm{http}: / / c r e a t i v e c o m m o n s . o r g / l i c e n s e s / b y / 4.0 /$, which permits unrestricted use, distribution, and reproduction in any medium, provided the original work is properly cited. Conditions, terms of use and publishing policy can be found at https://www.scienceopen.com/.

Preprint statement: This article is a preprint and has not been peer-reviewed, under consideration and submitted to ScienceOpen Preprints for open peer review.

DOI: 10.14293/S2199-1006.1.SOR-.PPBT1S7.v1

Preprint first posted online: 21 December 2020

Keywords: Unitary principle; Lorentz transformation; complete space-time transformation; constant speed of light in isolated reference frame; relative variable light speed 


\title{
On the Relativity of the Speed of Light
}

\author{
X. D. Dongfang \\ Wutong Mountain National Forest Park, Shenzhen, China
}

\begin{abstract}
Einstein's assumption that the speed of light is constant is a fundamental principle of modern physics with great influence. However, the nature of the principle of constant speed of light is rarely described in detail in the relevant literatures, which leads to a deep misunderstanding among some readers of special relativity. Here we introduce the unitary principle, which has a wide application prospect in the logic self consistency test of mathematics, natural science and social science. Based on this, we propose the complete space-time transformation including the Lorentz transformation, clarify the definition of relative velocity of light and the conclusion that the relative velocity of light is variable, and further prove that the relative variable light speed is compatible with Einstein's constant speed of light. The specific conclusion is that the propagation speed of light in vacuum relative to the observer's inertial reference frame is always constant $c$, but the propagation speed of light relative to any other inertial reference frame which has relative motion with the observer is not equal to the constant $c$; observing in all inertial frame of reference, the relative velocity of light propagating in the same direction in vacuum is 0 , while that of light propagating in the opposite direction is $2 c$. The essence of Einstein's constant speed of light is that the speed of light in an isolated reference frame is constant, but the relative speed of light in vacuum is variable. The assumption of constant speed of light in an isolated frame of reference and the inference of relative variable light speed can be derived from each other.
\end{abstract}

\section{INTRODUCTION}

Because of relativity, the speed of light in vacuum has a very special position in modern physics. Although the hypothesis of invariance of light speed $[1,2]$ and the principle of relativity[3], which are the basis of special relativity, are regarded as the two basic principles that can stand the test of experiment[4-10] and the demonstration of logical completeness[11-13], the essences of the hypothesis of constant speed of light and the inferences of Lorentz transformation are not always clear to all physics readers. This is because the relevant experimental results can be interpreted differently[14, 15]. Of course, the socalled superluminal and slow light speed phenomena[1624] are not variable light speed phenomena in the usual sense, while various superluminal and variable light speed theories[25-30] are only limited to formal reasoning.

The conclusions of theoretical physics often come from mathematical deduction. We know that from different angles, the same mathematical physics problem can have completely different formal solutions, and each formal solution can have different interpretations. Only the real solution of a mathematical physics problem conforms to the natural law, and the real solution of mathematical physics problem should be unique. If we discuss the transformation of time and space parameters from the perspective of the existence and uniqueness theorem for the solution of mathematical physics problems, the essences of the hypothesis of invariable light speed and the inference of Lorentz transformation will be clear. At this time, it is very important to put forward the relevant mathematical and physical problems. There is a universal principle to test the self consistency of natural science theory: different metrics can be used to describe natural laws, and there is a certain transformation relationship between them. The natural laws themselves do not change because of the different metrics. The results of the transformation from different mathematical form$s$ of natural laws expressed in different metrics into the same metric must be the same as the inherent form under this metric, $1=1$, which means the transformation is $u$ nitary. This principle is called the unitary principle. It can be widely used to test the self consistency of logic in mathematics, physics and even philosophy. By using the unitary principle to test the physical hypothesis and inference that can not be proved, the controversial problem can be transformed into the problem of definite solution of mathematical physics, so as to find the real solution of the problem under the condition of definite solution and distinguish it from the formal solution, and the problem will have a clear answer.

The unitary principle test of quantum mechanics has promoted the discovery of many important problems and conclusions from modern physics to com quantum theory[31-34]. In this paper, we use the unitary principle to test the concept of space-time in the sense of special relativity, and put forward the complete space-time transformation that includes the Lorentz transformation and thus conforms to the meaning of special relativity. We can find that the definition of relative speed of light exists, which is no longer constant. Furthermore, we can prove that the relative variable light speed is compatible with Einstein's constant speed of light.

\section{COMPLETE TRANSFORMATION OF TIME AND SPACE PARAMETERS}

Let the relative speed of two inertial reference frames $\Sigma$ and $\Sigma^{\prime}$ be $v$, and the unitary principle test of the logic self consistency of the hypothesis of constant light speed in vacuum contains the following four metrics: (a) the 
speed of light measured on reference frame $\Sigma$ relative to reference frame $\Sigma$; (b) the speed of light measured on reference frame $\Sigma^{\prime}$ relative to reference frame $\Sigma^{\prime}$; (c) the speed of light measured on reference frame $\Sigma$ relative to reference frame $\Sigma^{\prime}$; (d) the speed of light measured on reference frame $\Sigma^{\prime}$ relative to reference frame $\Sigma$. According to the unitary principle, the result of the transformation of light speed between different metric must be the same as the inherent speed of light under this metric, and the transformation is unitary.

It should be pointed out that the self consistent logic must satisfy the unitary principle, and the logic that violates the unitary principle must not be self consistent and implies many contradictions, while the physical logic with local self consistency may not be the natural law. Logical self consistency is only a necessary condition but not a sufficient condition for the theory of natural science. With regard to the space-time coordinates of the so-called event $P$, the concept of special relativity description is as follows:

(i) The time-space coordinate $(x, y, z, t)$ of event $P$ measured on reference frame $\Sigma$ relative to reference frame $\Sigma$;

(ii) The time-space coordinate $\left(x^{\prime}, y^{\prime}, z^{\prime}, t^{\prime}\right)$ of event $P$ measured on the reference frame $\Sigma^{\prime}$ relative to the reference frame $\Sigma^{\prime}$;

There are actually two other concepts:

(iii) The spatiotemporal coordinate $(\xi, \zeta, \eta, \tau)$ of event $P$ measured on reference frame $\Sigma$ relative to reference frame $\Sigma^{\prime}$;

(iv) The spatiotemporal coordinate $\left(\xi^{\prime}, \zeta^{\prime}, \eta^{\prime}, \tau^{\prime}\right)$ of event $P$ measured in the reference frame $\Sigma^{\prime}$ relative to the reference frame $\Sigma$.

It can be seen that for two inertial reference frames, an event has four sets of space-time concepts. The special theory of relativity based on the Lorentz transformation only describes the first two sets of space-time concepts $(x, y, z, t)$ and $\left(x^{\prime}, y^{\prime}, z^{\prime}, t^{\prime}\right)$.

On reference frame $\Sigma$, measuring the time and space parameters of event $P$ relative to reference frame $\Sigma$ and to reference frame $\Sigma^{\prime}$, the clock used is the clock on reference frame $\Sigma$, and the concept of time belongs to reference frame $\Sigma$, so $\tau=t$. Similarly, on reference frame $\Sigma^{\prime}$, measuring the time and space parameters of event $P$ relative to reference frame $\Sigma^{\prime}$ and relative reference frame $\Sigma$, the clock used is clock on reference frame $\Sigma^{\prime}$, and the concept of time belongs to the reference frame $\Sigma^{\prime}$, so $\tau^{\prime}=t^{\prime}$.

$$
\tau=t, \tau^{\prime}=t^{\prime}
$$

The above equations show the characteristics of isolated reference frame of time, that is, in all inertial reference frame, the observer can measure the motion law of an object relative to its own frame and relative to other inertial frames, but the clock used can only be the clock of that reference frame. The meaning of time here accords with Einstein's definition, that is, when the reading $t=$ $t^{\prime}=0$ of the calibrated synchronous clocks resting in the reference frame $\Sigma$ and $\Sigma^{\prime}$ respectively, the coordinate origin points of the two inertial frames coincide exactly.

There is a class of basic facts that have a potential impact on physical theory. For example, when the moon is on the line between the sun and the earth, measuring the distance between the sun and the earth on the earth can be divided into two parts: the distance between the sun and the moon and the distance between the moon and the earth; When the moon is not on the line between the sun and the earth, measuring the position vector of the sun relative to the earth on the earth can be decomposed into two parts: the position vector of the sun relative to the moon and the position vector of the moon relative to the earth. This kind of fact is abstracted as an axiom: the position vector of an object relative to other inertial reference frames in all inertial reference frame obeys the operational rule of vector superposition. We call it the principle of the relative position vector superposition.

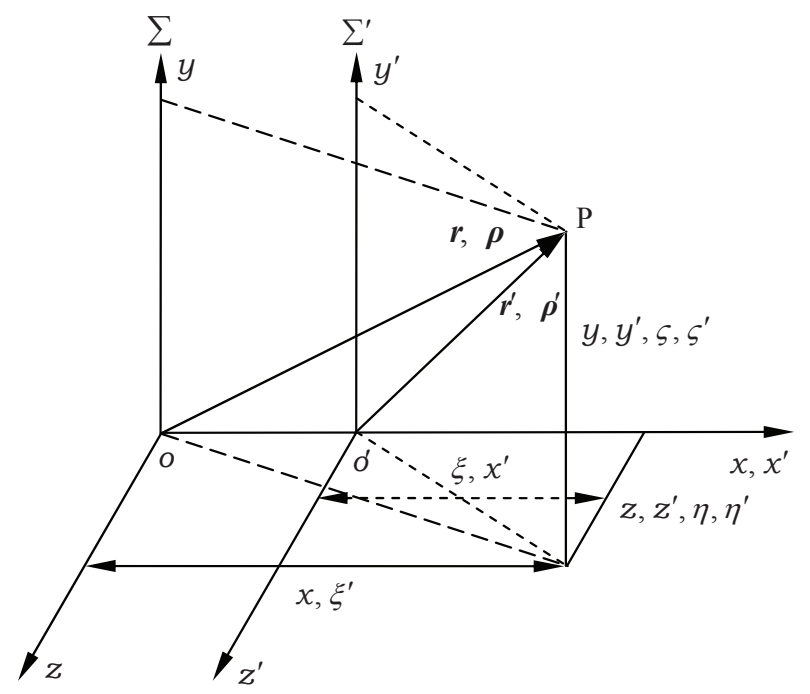

FIG. 1. Four sets of spatiotemporal concepts of the same event relative to two inertial reference frames

The above problems (iii) and (iv) can now be solved. As shown in Fig. 1, according to the agreement of special relativity, the two inertial reference frames $\Sigma$ and $\Sigma^{\prime}$ have a common $x$ axis. When the moment of reference frame $\Sigma$ is $t$, the distance between the coordinate origins of the two coordinate frames is $\overline{o o^{\prime}}=v t$. Since the distance between an object measured in all inertial reference frame relative to other inertial frames can be divided, then $x=\overline{o o^{\prime}}=v t+\xi$, therefore $\xi=x-v t$. Because $\zeta=y, \eta=z$, then the transformation relationship between the spatiotemporal coordinates $(x, y, z, t)$ relative to reference frame $\Sigma$ and the spatiotemporal coordinates $(\xi, \zeta, \eta, \tau)$ relative to reference frame $\Sigma^{\prime}$ of event 
$P$ measured in reference frame $\Sigma$ is as follows

$$
\xi=x-v t, \zeta=y, \eta=z, \tau=t
$$

Similarly, observed in the reference frame $\Sigma^{\prime}$, the transformation relationship between the spatiotemporal coordinate $\left(x^{\prime}, y^{\prime}, z^{\prime}, t^{\prime}\right)$ of event $P$ at time $t^{\prime}$ relative to the reference frame $\Sigma^{\prime}$ and the spatiotemporal coordinate $\left(\xi^{\prime}, \zeta^{\prime}, \eta^{\prime}, \tau^{\prime}\right)$ of the relative reference frame $\Sigma$ is as follows

$$
\xi^{\prime}=x^{\prime}+v t^{\prime}, \zeta^{\prime}=y^{\prime}, \eta^{\prime}=z^{\prime}, \tau^{\prime}=t^{\prime}
$$

The meaning of Einstein's constant speed of light hypothesis is that the speed of the observed light relative to the reference frame in any inertial frame is $c$, which is expressed as $|d \mathbf{r} / d t|=c,\left|d \mathbf{r}^{\prime} / d t^{\prime}\right|=c$. Therefore, the invariance of light speed in special relativity belongs to the constant speed of light in an isolated reference frame. Einstein derived Lorentz transformation according to the principle of constant light speed in isolated reference frame and relativity principle

$$
x^{\prime}=\frac{x-v t}{\sqrt{1-v^{2} c^{-2}}}, y^{\prime}=y, z^{\prime}=z, t^{\prime}=\frac{t-v c^{-2} x}{\sqrt{1-v^{2} c^{-2}}}
$$

The inverse transformation is

$$
x=\frac{x^{\prime}+v t^{\prime}}{\sqrt{1-v^{2} c^{-2}}}, y=y^{\prime}, z=z^{\prime}, t=\frac{t^{\prime}+v c^{-2} x^{\prime}}{\sqrt{1-v^{2} c^{-2}}}
$$

Obviously, Lorentz transformation in the sense of special relativity describes the relationship between space-time parameters of isolated reference frame between two inertial reference frames.

Now we can determine the relations among various defined spatial parameters. By substituting the first formula of the set of relations (2) into the first formula of Lorentz transformation (4), or the first formula of the set of relations (3) into the first formula of Lorentz inverse transformation (5), new transformation and corresponding inverse transformation are obtained respectively

$$
x^{\prime}=\frac{\xi}{\sqrt{1-v^{2} c^{-2}}}, x=\frac{\xi^{\prime}}{\sqrt{1-v^{2} c^{-2}}}
$$

After substituting the equivalent form $x^{\prime}=\xi^{\prime}-v t^{\prime}$ of the first formula of (3) into the first formula of formula (6), or substituting the equivalent form $x=\xi+v t$ of the first formula of (2) into the second formula of formula (6), we get the following results

$$
\xi=\sqrt{1-v^{2} c^{-2}}\left(\xi^{\prime}-v t^{\prime}\right), \xi^{\prime}=\sqrt{1-v^{2} c^{-2}}(\xi+v t)
$$

These transformations are different from both Lorentz transformation and Galileo transformation.

Equations (2), (3), (4) and (5) describe the transformation relations among four groups of space-time parameters of two inertial reference frames with relative velocity $v$. The form of the equations is as follows,

$$
\left\{\begin{array} { l } 
{ x ^ { \prime } = \frac { x - v t } { \sqrt { 1 - v ^ { 2 } c ^ { - 2 } } } } \\
{ y ^ { \prime } = y = \zeta = \zeta ^ { \prime } } \\
{ z ^ { \prime } = z = \eta = \eta ^ { \prime } } \\
{ t ^ { \prime } = \frac { t - v c ^ { - 2 } x } { \sqrt { 1 - v ^ { 2 } c ^ { - 2 } } } }
\end{array} \Leftrightarrow \left\{\begin{array}{l}
x^{\prime}=\frac{\xi}{\sqrt{1-v^{2} c^{-2}}} \\
\xi^{\prime}=x^{\prime}+v t^{\prime} \\
\tau=t, \tau^{\prime}=t^{\prime} \\
\xi^{\prime}=\sqrt{1-v^{2} c^{-2}}(\xi+v t)
\end{array}\right.\right.
$$

The corresponding inverse transformation relation is

$$
\left\{\begin{array} { l } 
{ x = \frac { x ^ { \prime } + v t ^ { \prime } } { \sqrt { 1 - v ^ { 2 } c ^ { - 2 } } } } \\
{ y = y ^ { \prime } = \zeta ^ { \prime } = \zeta ^ { \prime } } \\
{ z = z ^ { \prime } = \eta ^ { \prime } = \eta } \\
{ t = \frac { t ^ { \prime } + v c ^ { - 2 } x ^ { \prime } } { \sqrt { 1 - v ^ { 2 } c ^ { - 2 } } } }
\end{array} \Leftrightarrow \left\{\begin{array}{l}
x=\frac{\xi^{\prime}}{\sqrt{1-v^{2} c^{-2}}} \\
\xi=x-v t \\
t=\tau, \tau^{\prime}=t^{\prime} \\
\xi=\sqrt{1-v^{2} c^{-2}}\left(\xi^{\prime}-v t^{\prime}\right)
\end{array}\right.\right.
$$

The above transformation relations (8) and (9) conform to the special relativity meaning, and are called complete space-time transformation. According to the complete space-time transformation, the complete transformation relations between the intrinsic quantity and the relative quantity of the corresponding physical quantity such as momentum and energy between different inertial reference frames can be derived, which is omitted here. We also need to give priority to discovering the deeper spatiotemporal parameter transformations

\section{VARIABILITY OF RELATIVE SPEED OF LIGHT}

The Lorentz transformation is essentially a speed preserving transformation, which stipulates that the speed of light in the vacuum of two isolated reference frames is constant, thus defining the transformation relationship of the space-time parameters of the two inertial reference frames. Since the speed of the experimental observation light relative to the laboratory reference frame in the vacuum is constant $c$, Einstein proposed the hypothesis that the speed of light does not change. In turning, he derived the Lorentz transformation and established special relativity[35]. Now that we have a complete space-time transformation, we have a clear definition of the relative speed of light. According to the complete space-time transformation, the relative speed of light is variable, and the relative variable light speed is compatible with the constant speed of light in an isolated reference frame.

It is assumed that when the coordinate origin $o$ of the inertial reference frame $\Sigma$ coincides with the coordinate origin $o^{\prime}$ of the inertial reference frame $\Sigma^{\prime}$, the light source at the common origin emits a photon to the positive direction of the $x$ axis. According to the complete spacetime transformation, the two inertial frames of reference contain four definitions of light speed, namely: 
a) The isolated speed of the photon relative to the reference frame $\Sigma$ measured on reference frame $\Sigma$, $\left|\frac{d \mathbf{r}}{d t}\right|=\sqrt{\left(\frac{d x}{d t}\right)^{2}+\left(\frac{d y}{d t}\right)^{2}+\left(\frac{d z}{d t}\right)^{2}}=c ;$

b) The relative light speed of the photon relative to $\Sigma^{\prime}$ measured on reference frame $\Sigma,\left|\frac{d \rho}{d \tau}\right|=$ ?;

c) The isolated speed of the photons relative to reference frame $\Sigma^{\prime}$ measured on reference frame $\Sigma^{\prime}$, $\left|\frac{d \mathbf{r}^{\prime}}{d t^{\prime}}\right|=\sqrt{\left(\frac{d x^{\prime}}{d t^{\prime}}\right)^{2}+\left(\frac{d y^{\prime}}{d t^{\prime}}\right)^{2}+\left(\frac{d z^{\prime}}{d t^{\prime}}\right)^{2}}=c ;$

d) The relative light speed of the photon relative to $\Sigma$ measured on reference frame $\Sigma^{\prime},\left|\frac{d \rho^{\prime}}{d \tau^{\prime}}\right|=$ ?

The constant speed of light in an isolated frame of reference is well known, and the results are written directly above.

Now let's calculate the two relative speeds of light $\left|\frac{d \rho}{d \tau}\right|$ and $\left|\frac{d \rho^{\prime}}{d \tau^{\prime}}\right|$. According to the transformation formula (2), $\frac{d \xi}{d \tau}=\frac{d(x-v t)}{d t}=\frac{d x}{d t}-v, \frac{d \zeta}{d \tau}=\frac{d y}{d t}$ and $\frac{d \eta}{d \tau}=\frac{d z}{d t}$ are obtained. The isolated light speed $\left|\frac{d \mathbf{r}}{d t}\right|=\sqrt{\left(\frac{d x}{d t}\right)^{2}+\left(\frac{d y}{d t}\right)^{2}+\left(\frac{d z}{d t}\right)^{2}}=$ $c$ on the reference frame $\Sigma$ will be used in the calculation. Therefore, the results of the relative light velocity $\frac{d \rho}{d \tau}$ of photons relative to the reference frame $\Sigma^{\prime}$ measured on the reference frame $\Sigma$ satisfy the relationship

$$
\begin{aligned}
& \left|\frac{d \rho}{d \tau}\right|=\sqrt{\left(\frac{d \xi}{d \tau}\right)^{2}+\left(\frac{d \varsigma}{d \tau}\right)^{2}+\left(\frac{d \eta}{d \tau}\right)^{2}} \\
& =\sqrt{\left(\frac{d x}{d t}-v\right)^{2}+\left(\frac{d y}{d t}\right)^{2}+\left(\frac{d z}{d t}\right)^{2}} \\
& =\sqrt{\left(\frac{d x}{d t}\right)^{2}+\left(\frac{d y}{d t}\right)^{2}+\left(\frac{d z}{d t}\right)^{2}-2 \frac{d x}{d t} v+v^{2}} \\
& =\sqrt{c^{2}-2 v \frac{d x}{d t}+v^{2}} \neq c
\end{aligned}
$$

If the light propagates along the common $x$ axis of the two inertial reference frames, that is, the velocity of light relative to the reference frame $\Sigma$ measured on the reference frame $\Sigma$ is $\frac{d x}{d t}= \pm c, \frac{d y}{d t}=0$ and $\frac{d z}{d t}=0$, then the speed of light relative to the reference frame $\Sigma^{\prime}$ measured on the reference frame $\Sigma$ is,

$$
\left|\frac{d \rho}{d \tau}\right|=|c \mp v|
$$

If the reference frame $\Sigma^{\prime}$ is also a photon, $v= \pm c$. Therefore, the relative velocity of light propagating in the same direction is 0 while that of light propagating in the opposite direction is $2 c$ measured on the reference frame $\Sigma$.

Similarly, according to the transformation formula (3), $\frac{d \xi^{\prime}}{d t^{\prime}}=\frac{d\left(x^{\prime}+v t^{\prime}\right)}{d t^{\prime}}=\frac{d x^{\prime}}{d t^{\prime}}+v, \frac{d \zeta^{\prime}}{d \tau^{\prime}}=\frac{d y^{\prime}}{d t^{\prime}}$ and $\frac{d \eta^{\prime}}{d \tau^{\prime}}=\frac{d z^{\prime}}{d t^{\prime}}$ are obtained. In the calculation, the isolated light speed $\left|\frac{d \mathbf{r}^{\prime}}{d t^{\prime}}\right|=\sqrt{\left(\frac{d x^{\prime}}{d t^{\prime}}\right)^{2}+\left(\frac{d y^{\prime}}{d t^{\prime}}\right)^{2}+\left(\frac{d z^{\prime}}{d t^{\prime}}\right)^{2}}=c$ of the reference frame $\Sigma^{\prime}$ is used again, so the results of the relative light velocity $\frac{d \rho^{\prime}}{d \tau^{\prime}}$ of photons relative to the reference frame $\Sigma$ measured in the reference frame $\Sigma^{\prime}$ satisfy the relationship

$$
\begin{aligned}
& \left|\frac{d \rho^{\prime}}{d \tau^{\prime}}\right|=\sqrt{\left(\frac{d \xi^{\prime}}{d \tau^{\prime}}\right)^{2}+\left(\frac{d \varsigma^{\prime}}{d \tau^{\prime}}\right)^{2}+\left(\frac{d \eta^{\prime}}{d \tau^{\prime}}\right)^{2}} \\
& =\sqrt{\left(\frac{d x^{\prime}}{d t^{\prime}}+v\right)^{2}+\left(\frac{d y^{\prime}}{d t^{\prime}}\right)^{2}+\left(\frac{d z^{\prime}}{d t^{\prime}}\right)^{2}} \\
& =\sqrt{\left(\frac{d x^{\prime}}{d t^{\prime}}\right)^{2}+\left(\frac{d y^{\prime}}{d t^{\prime}}\right)^{2}+\left(\frac{d z^{\prime}}{d t^{\prime}}\right)^{2}+2 \frac{d x^{\prime}}{d t^{\prime}} v+v^{2}} \\
& =\sqrt{c^{2}+2 v \frac{d x^{\prime}}{d t^{\prime}}+v^{2}} \neq c
\end{aligned}
$$

For the light propagating along the common $x$ axis of the two inertial reference frames, the velocity of the light measured on the reference frame $\Sigma^{\prime}$ relative to the reference frame $\Sigma^{\prime}$ is $\frac{d x^{\prime}}{d t^{\prime}}= \pm c, \frac{d y^{\prime}}{d t^{\prime}}=0$ and $\frac{d z^{\prime}}{d t^{\prime}}=0$, while the speed of the light measured on the reference frame $\Sigma^{\prime}$ relative to the reference frame $\Sigma$ is

$$
\left|\frac{d \rho^{\prime}}{d \tau^{\prime}}\right|=|c \pm v|
$$

If the reference frame $\Sigma$ is also a photon, $v= \pm c$. Therefore, the relative velocity of light propagating in the same direction is 0 while that of light propagating in the opposite direction is $2 c$ measured on the reference frame $\Sigma$.

Formulas (10) and (13) show that when the velocity between two inertial reference frames is not zero, the two relative light velocities are related to the relative velocities of inertial reference frames. Obviously, the relative velocity of light propagating in the same direction is 0 and that of light propagating in the opposite direction is $2 c$ when observed in any inertial reference. Under the complete space-time transformation, the relative speed of light is variable, and the Einstein's constant speed of light in isolated frame of reference is a necessary condition for the relative variable speed of light.

\section{COMPATIBILITY BETWEEN RELATIVE LIGHT SPEED AND SOLITARY LIGHT SPEED}

Now we know that Einstein's constant speed of light hypothesis means that the speed of light in an isolated frame of reference is constant, while the relative speed of light is variable. The unitary principle requires that the result of the transformation of the relative speed of light in vacuum into the isolated speed of light in vacuum must be the constant $c$, otherwise it will constitute a negative result of $1 \neq 1$. Therefore, the constant Einstein speed 
of light must also be able to be derived from the variable relative speed of light. The proof is as follows.

Relationships $\frac{d x}{d t}=\frac{d \xi}{d t}+v=\frac{d \xi}{d \tau}+v, \frac{d y}{d t}=\frac{d \varsigma}{d t}=\frac{d \varsigma}{d \tau}$ and $\frac{d z}{d t}=\frac{d \eta}{d t}=\frac{d \eta}{d \tau}$ are obtained from the complete inverse spacetime transformation (9). First, substituting theses relationships into the calculation formula $\left|\frac{d \mathbf{r}}{d t}\right|=$ $\sqrt{\left(\frac{d x}{d t}\right)^{2}+\left(\frac{d y}{d t}\right)^{2}+\left(\frac{d z}{d t}\right)^{2}}$ of the speed in isolated reference frame of $\sum$, and then using relative light speed $\left|\frac{d \rho}{d \tau}\right|^{2}=\left(\frac{d \xi}{d \tau}\right)^{2}+\left(\frac{d \varsigma}{d \tau}\right)^{2}+\left(\frac{d \eta}{d \tau}\right)^{2}=c^{2}-2 v \frac{d x}{d t}+v^{2}$ given in equation (10), we have

$$
\begin{aligned}
& \left|\frac{d \mathbf{r}}{d t}\right|=\sqrt{\left(\frac{d \xi}{d \tau}+v\right)^{2}+\left(\frac{d \varsigma}{d \tau}\right)^{2}+\left(\frac{d \eta}{d \tau}\right)^{2}} \\
& =\sqrt{\left(\frac{d \xi}{d \tau}\right)^{2}+\left(\frac{d \varsigma}{d \tau}\right)^{2}+\left(\frac{d \eta}{d \tau}\right)^{2}+2 v \frac{d \xi}{d \tau}+v^{2}} \\
& =\sqrt{c^{2}-2 v \frac{d x}{d t}+2 v \frac{d \xi}{d \tau}+2 v^{2}} \\
& =\sqrt{c^{2}-2 v\left(\frac{d \xi}{d \tau}+v\right)+2 v \frac{d \xi}{d \tau}+2 v^{2}}=c
\end{aligned}
$$

Similarly, relationships $\frac{d x^{\prime}}{d t^{\prime}}=\frac{d \xi^{\prime}}{d t^{\prime}}-v=\frac{d \xi^{\prime}}{d \tau^{\prime}}-v, \frac{d y^{\prime}}{d t^{\prime}}=\frac{d \varsigma^{\prime}}{d t^{\prime}}=$ $\frac{d \varsigma^{\prime}}{d \tau^{\prime}}$ and $\frac{d z^{\prime}}{d t^{\prime}}=\frac{d \eta^{\prime}}{d t^{\prime}}=\frac{d \eta^{\prime}}{d \tau^{\prime}}$ are obtained from the complete inverse spacetime transformation (8). By substituting theses relationships into the into the calculation formula $\left|\frac{d \mathbf{r}^{\prime}}{d t^{\prime}}\right|=\left(\frac{d x^{\prime}}{d t^{\prime}}\right)^{2}+\left(\frac{d y^{\prime}}{d t^{\prime}}\right)^{2}+\left(\frac{d z^{\prime}}{d t^{\prime}}\right)^{2}$ of the speed in isolated reference frame of $\sum^{\prime}$, and using again the relative light speed $\left|\frac{d \rho^{\prime}}{d \tau^{\prime}}\right|^{2}=\left(\frac{d \xi^{\prime}}{d \tau^{\prime}}\right)^{2}+\left(\frac{d \varsigma^{\prime}}{d \tau^{\prime}}\right)^{2}+\left(\frac{d \eta^{\prime}}{d \tau^{\prime}}\right)^{2}=c^{2}+2 v \frac{d x^{\prime}}{d t^{\prime}}+v^{2}$ given by formula (12), the following result is obtained,

$$
\begin{aligned}
& \left|\frac{d \mathbf{r}^{\prime}}{d t^{\prime}}\right|=\sqrt{\left(\frac{d \xi^{\prime}}{d \tau^{\prime}}-v\right)^{2}+\left(\frac{d \varsigma^{\prime}}{d \tau^{\prime}}\right)^{2}+\left(\frac{d \eta^{\prime}}{d \tau^{\prime}}\right)^{2}} \\
& =\sqrt{\left(\frac{d \xi^{\prime}}{d \tau^{\prime}}\right)^{2}+\left(\frac{d \varsigma^{\prime}}{d \tau^{\prime}}\right)^{2}+\left(\frac{d \eta^{\prime}}{d \tau^{\prime}}\right)^{2}-2 v \frac{d \xi^{\prime}}{d \tau^{\prime}}+v^{2}} \\
& =\sqrt{c^{2}+2 v \frac{d x^{\prime}}{d t^{\prime}}-2 v \frac{d \xi^{\prime}}{d \tau^{\prime}}+2 v^{2}} \\
& =\sqrt{c^{2}+2 v\left(\frac{d \xi^{\prime}}{d \tau^{\prime}}-v\right)-2 v \frac{d \xi^{\prime}}{d \tau^{\prime}}+2 v^{2}}=c
\end{aligned}
$$

It can be seen that according to relative light speed in the complete space-time transformation, constant light speed in isolated reference frame can be derived. It can be said that the relative variable light speed is also a necessary condition for constant light speed in isolated reference frame.

The calculation results of (10) - (15) above show that there are definitions of constant solitary light speed and variable relative light speed under the complete spacetime transformation. Einstein's invariance of light speed belongs to the constant speed of light in isolated reference frame. The constant speed of light in isolated frame of reference is compatible with the relative variable speed of light, and they do not constitute a seemingly opposite negative basis on the surface. All these calculations are elementary, but elementary does not mean that the corresponding problems and conclusions are of little importance. Often, the discovery of primary problems is more difficult than the discovery of higher problems, and the impact of primary problems is more profound.

\section{CONCLUSIONS AND COMMENTS}

This paper introduces the unitary principle, which has a wide application prospect. Then, the space-time transformation of special relativity is tested by the unitary principle, and the complete space-time transformation is proposed. It is shown that the relative variable speed of light is compatible with Einstein's hypothesis of constant speed of light. Einstein's invariable speed of light belongs to the constant speed of light in isolated reference frame, that is, the speed of light in vacuum relative to that reference frame measured on any isolated inertial reference frame is $c$, which is the real meaning of constant light speed. In fact, the concept of "event" in special relativity defines the characteristics of isolated reference frame of space and time concepts used to describe physical laws. The mapping relationship between space-time concepts of each isolated reference frame is determined by the Lorentz transformation. From the laboratory reference system, if the light moves in the opposite direction with other reference systems, the vacuum light speed relative to that reference frame is greater than $c$; if the light moves in the same direction with other reference system$\mathrm{s}$, the vacuum light speed relative to that reference frame is less than $c$.

The condition of an inertial reference frame is very special because there is no real inertial reference frame in nature. Even if the inertial reference frame conditions are satisfied, if the speed of a particle relative to other inertial reference systems measured in the laboratory reference frame is greater than the speed of light in vacuum, the isolated speed of a particle measured on that inertial reference frame will always be less than the speed of light in vacuum. This is the essence of the speed limit of special relativity. The Lorentz transformation determines that the velocity of the isolated reference frame of matter motion is less than that of light in vacuum. However, this speed limit theory does not apply to relative velocity. The observer cannot stand on photons, and the relative velocity of light to light in the vacuum observed in the laboratory is actually distributed between 0 and $2 c$. It should be stated again that the length of an object measured in the same inertial frame is separable, that is, the length of straight lines observed in all inertial reference frame can be added or subtracted. In a broad sense, the position vectors between moving objects observed in the same inertial frame obey the rule of vector superposition. 
Because of this, if the relative variable speed of light is negated, the constant speed of light in Einstein's isolated reference frame is also denied. However, there may be no causal relationship between the relative variable speed of light and the constant speed of light in an isolated reference frame, although the relative variable speed of light and the constant speed of light in isolated reference frame can be derived from each other in the sense of complete space-time transformation.
The complete space-time transformation based on the unitary principle solves the doubts caused by the invariable speed of light. However, the relationship between it and the laws of nature needs to be further revealed. The unitary principle can be widely used in logic self consistency test, so as to effectively solve those tangled logical problems, and will play a more important role in the future new scientific theory.
[1] Nerlich, G. Special relativity is not based on causality. The British Journal for the Philosophy of Science 33, 361-388 (1982).

[2] Garrison, J., Mitchell, M., Chiao, R. \& Bolda, E. Superluminal signals: causal loop paradoxes revisited. Physics Letters A 245, 19-25 (1998).

[3] Einstein, A. Relativity: The Special and the General Theory-100th Anniversary Edition. (Princeton University Press, 2019).

[4] Michelson, A. A. ART. XXI. The relative motion of the Earth and the Luminiferous ether. American Journal of Science (1880-1910) 22, 120 (1881).

[5] Müller, H., Herrmann, S., Braxmaier, C., Schiller, S. \& Peters, A. Modern Michelson-Morley Experiment using Cryogenic Optical Resonators. Physical Review Letters 91, 020401 (2003).

[6] Müller, H et al. Tests of Relativity by Complementary Rotating Michelson-Morley Experiments. Physical Review Letters 99, 50401 (2007).

[7] Haugan, M. P. and Will, C. M. Modern tests of special relativity. Phys. Today 40, 69 (1987).

[8] Zhang, Y. Z. Special relativity and its experimental foundations. (World Scientific, 1997).

[9] Antonini, P., Okhapkin, M., G kl? E. \& Schiller, S. Test of constancy of speed of light with rotating cryogenic optical resonators. Physical Review A 71, 50101 (2005).

[10] Magueijo, J. New varying speed of light theories. Reports on Progress in Physics 66, 2025 (2003).

[11] Mansouri, R. and Sexl, R. U. A test theory of special relativity: I. Simultaneity and clock synchronization. General relativity and Gravitation 8, 497 (1977).

[12] Mansouri, R. \& Sexl, R. U. A test theory of special relativity: III. Second-order tests. General relativity and Gravitation 8, 809-814 (1977).

[13] Will, C. M. Theory and experiment in gravitational physics. (Cambridge university press, 2018).

[14] Clément, G. Does the Fizeau experiment really test special relativity? American Journal of Physics 48, 1059 (1980).

[15] Wang, L-J., Liu, N., Lin, Q. \& Zhu, S. Superluminal propagation of light pulses: A result of interference. Physical Review E 68, 66606 (2003).

[16] Alväger, T., Kreisler, M. Quest for faster-than-light particles. Physical Review 171, 1357-1361 (1968).

[17] Parker, L. Faster-Than-Light Intertial Frames and Tachyons. Physical Review 188, 2287-2292 (1969).

[18] Wang, L-J., Kuzmich, A. \& Dogariu, A. Gain-assisted superluminal light propagation. Nature 406, 277-279 (2000).

[19] Bigelow, M., Lepeshkin, N. \& Boyd, R. Superluminal and slow light propagation in a room-temperature solid, American Association for the Advancement of Science, 301, 200-202 (2003).

[20] Bortman-Arbiv, D., Wilson-Gordon, A. \& Friedmann, H. Phase control of group velocity: From subluminal to superluminal light propagation. Physical Review A 63, 43818 (2001).

[21] Kang, H., Hernandez, G. \& Zhu, Y. Superluminal and slow light propagation in cold atoms. Physical Review A 70, 11801 (2004).

[22] Bo, F., Zhang, G. \& Xu, J. Transition between superluminal and subluminal light propagation in photorefractive $\mathrm{Bi} 12 \mathrm{SiO} 20$ crystals. Phys. Rev. A 64, 053809 (2001).

[23] Sun, H. et al. Light propagation from subluminal to superluminal in a three-level $\Lambda$-type system. Physics Letters A 335, 68-75 (2005).

[24] Salart, D., Baas, A., Branciard, C., Gisin, N. \& Zbinden, $\mathrm{H}$. Testing the speed of 'spooky action at a distance'. Nature 454, 861-864 (2008).

[25] Recami, E. How to recover causality in special relativity for tachyons. Foundations of Physics (Historical Archive) 8, 329-340 (1978).

[26] Schwartz, C. Some improvements in the theory of fasterthan-light particles. Physical Review D 25, 356-364 (1982).

[27] Gonzalez-Mestres, L. Cosmological implications of a possible class of particles able to travel faster than light. Nucl. Phys. B, Proc. Suppl. 48 (1996).

[28] Everett, A. Tachyons, broken Lorentz invariance, and a penetrable light barrier. Physical Review D 13, 785-794 (1976).

[29] Pant, M., Bisht, P., Negi, O. \& Rajput, B. Dirac spinors and tachyon quantization. Canadian Journal of Physics 78, 303-315 (2000).

[30] Milonni, P., Furuya, K. \& Chiao, R. Quantum theory of superluminal pulse propagation. Phys. Rev 52, 1525 (1995).

[31] Chen, R. New Exact Solution of Dirac-Coulomb Equation with Exact Boundary Condition International Journal of Theoretical Physics 47 (2008) 881-890.

[32] Chen, R. Real Solution of Dirac Equation. arXiv:0908.4320 (physics.gen-ph, 2009).

[33] Dongfang, X. D. Com Quantum Theory, ResearchGate, the update will be persistent.

[34] Dongang, X. D. The Morbid Equation of Quantum Numbers, ResearchGate, 2019.

[35] Einstein, A. On the electrodynamics of moving bodies. Annalen der physik 17, 891-921 (1905). 